\title{
A hadronic minute-scale GeV flare from quasar 3C 279?
}

\author{
M. Petropoulou ${ }^{1 \star}$, K. Nalewajko², M. Hayashida ${ }^{3}$, A. Mastichiadis ${ }^{4}$ \\ ${ }^{1}$ Department of Physics and Astronomy, Purdue University, 525 Northwestern Avenue, West Lafayette, IN 47907, USA \\ ${ }^{2}$ Nicolaus Copernicus Astronomical Center, Polish Academy of Sciences ,ul. Bartycka 18, 00-716 Warszawa, Poland \\ ${ }^{3}$ Institute for Cosmic-Ray Research, University of Tokyo, 5-1-5 Kashiwanoha, Kashiwa, Chiba, 277-8582, Japan \\ ${ }^{4}$ Department of Physics, National and Kapodistrian University of Athens, Panepistimiopolis, GR 15783 Zografos, Greece
}

Received / Accepted

\begin{abstract}
The flat spectrum radio quasar 3C 279 is a known $\gamma$-ray variable source that has recently exhibited minute-scale variability at energies $>100 \mathrm{MeV}$. One-zone leptonic models for blazar emission are severely constrained by the short timescale variability that implies a very compact emission region at a distance of hundreds of Schwarzschild radii from the central black hole. Here, we investigate a hadronic scenario where $\mathrm{GeV} \gamma$-rays are produced via proton synchrotron radiation. We also take into account the effects of the hadronically initiated electromagnetic cascades (EMC). For a $\gamma$-ray emitting region in rough equipartition between particles and kG magnetic fields, located within the broad-line region (BLR), the development of EMC redistributes the $\gamma$-ray luminosity to softer energy bands and eventually leads to broadband spectra that differ from the observed ones. Suppression of EMC and energy equipartition are still possible, if the $\gamma$-ray emitting region is located beyond the BLR, is fast moving with Doppler factor $(>70)$, and contains strong magnetic fields $(>100 \mathrm{G})$. Yet, these conditions cannot be easily met in parsec-scale jets, thus disfavouring a proton synchrotron origin of the Fermi-LAT flare.
\end{abstract}

Key words: galaxies: active: individual: 3C 279 - gamma-rays: galaxies - radiation mechanisms: non-thermal

\section{INTRODUCTION}

Variability ranging from few hours to several weeks has been commonly observed at various energy bands of the blazar spectrum. What came as a surprise was the detection of short (few minutes) timescale variability at very high energy $\gamma$-rays (VHE, $E_{\gamma}>100 \mathrm{GeV}$ ) from several blazars, including BL Lac objects [Mrk 421 (Fortson et al. 2012), Mrk 501 (Albert et al. 2007), and PKS 2155-304 (Aharonian et al. 2007)] and flat spectrum radio quasars (FSRQ) (PKS 1222+216, Aleksić et al. 2011). The latest addition to the above list is the minute-scale flare detected on June 2015 at GeV $\gamma$-rays with Fermi-LAT (Ackermann et al. 2016) from FSRQ 3C 279 (at redshift z=0.536, Lynds et al. 1965).

The 2015 Fermi-LAT flare was characterized by high apparent $\gamma$-ray luminosity $\left(L_{\gamma} \sim 10^{49} \mathrm{erg} \mathrm{s}^{-1}\right)$, short flux-doubling timescales ( $\lesssim 5$ minutes), and high values of the Compton dominance parameter $(\kappa=100)$. The observed minute-scale variability suggests a very compact emission region at a distance of hundreds of Schwarzschild radii from the central black hole. In leptonic external Compton models (ECS) (Dermer et al. 1992; Sikora et al. 1994), the observed high Compton dominance requires a strongly matter-dominated emitting region (see also Asano \& Hayashida 2015). Models invoking the presence of relativistic protons in

\footnotetext{
^ Einstein Fellow; E-mail: mpetropo@purdue.edu
}

the jet have been proposed as an alternative to the ECS scenario. In these models, $\gamma$-rays may result from a hadronically initiated electromagnetic cascade (EMC) (Mannheim et al. 1991) or from relativistic proton synchrotron radiation (Aharonian 2000; Mücke \& Protheroe 2001). The efficiencies of both hadronic processes are expected to be enhanced at sub-parsec dissipation distances (see Khangulyan et al. 2013, for 3C 454.3).

In this letter, we investigate if a proton synchrotron origin of the $\mathrm{GeV}$ flare is possible. The leptohadronic synchrotron model (LHS) for blazars is often criticized for its energetic requirements (e.g. Zdziarski \& Böttcher 2015). Recently, Petropoulou \& Dermer (2016) - henceforth PD16, showed that the LHS model of the VHE blazar radiation can have sub-Eddington absolute jet powers (Cerruti et al. 2015), whereas models of dominant $>100 \mathrm{MeV}$ radiation in FSRQ may still require excessive power (for 3C 273, see Petropoulou \& Dimitrakoudis 2015). We do not discuss a photomeson ( $\mathrm{p} \gamma$ ) origin of the flare here, as this would have, in principle, higher energetic requirements than the LHS model (for Mrk 421, see Dimitrakoudis et al. 2014), and would result in flatter Xray-to- $\gamma$-ray spectra than observed; a detailed calculation will be presented elsewhere.

As a starting point, we seek for parameters that minimize the jet power and provide a successful fit to the time-averaged flare's spectrum from Orbit D (Ackermann et al. 2016). To do so, we use the analytical expressions presented in PD16 and the main observ- 
Table 1. Observables of the minute-scale GeV flare from 3C 279 used in our analysis. Parameters describing the broad line region (BLR) are also listed.

\begin{tabular}{llc}
\hline Parameter & Symbol & Value \\
\hline Variability timescale $(\mathrm{s})$ & $t_{\mathrm{V}}$ & 450 \\
$\gamma$-ray luminosity $\left(\mathrm{erg} \mathrm{s}^{-1}\right)$ & $L_{\gamma}$ & $10^{49}$ \\
$\gamma$-ray photon energy $(\mathrm{GeV})$ & $E_{\gamma}$ & 0.7 \\
IR-to-UV luminosity $\left(\mathrm{erg} \mathrm{s}^{-1}\right)$ & $L$ & $10^{47}$ \\
Compton dominance parameter & $\kappa$ & 100 \\
BLR luminosity ${ }^{\dagger}\left(\mathrm{erg} \mathrm{s}^{-1}\right)$ & $L_{\mathrm{BLR}}$ & $6 \times 10^{44}$ \\
BLR radius $^{\ddagger}(\mathrm{cm})$ & $R_{\mathrm{BLR}}$ & $2.5 \times 10^{17}$ \\
\hline
\end{tabular}

$\dagger$ This has been estimated using the observed broad emission lines (Celotti et al. 1997).

\#stimated using the scaling relation $R_{\mathrm{BLR}} \approx 10^{17} L_{\mathrm{d}, 45}^{1 / 2}$ (Ghisellini \& Tavecchio 2008).

ables of the flare (variability timescale $t_{\mathrm{v}}, \gamma$-ray luminosity $L_{\gamma}$, and photon energy $E_{\gamma}$ ). It is possible that the produced $\gamma$-rays do not escape but they are absorbed by the softer photons in the source initiating EMC cascades which alter drastically the broad-band spectrum as they distribute the $\gamma$-ray energy to lower frequencies, thus destroying the attempted spectral fit. To assess the role of EMC, we derive analytical expressions for the optical depth for internal photon-photon $(\gamma \gamma)$ absorption and the efficiency for $\mathrm{p} \gamma$ interactions. The parameter values derived in the first step are then used as an input to a numerical code that calculates the broadband photon spectrum taking into account all relevant physical processes (Dimitrakoudis et al. 2012). In our numerical investigation, we compare the broad-band photon spectra obtained with and without internal $\gamma \gamma$ absorption. If, for parameters that minimize the jet power, the absorption of VHE radiation produced via $\mathrm{p} \gamma$ interactions initiates an EMC with a spectrum that does not describe well the data, we search for other parameters that may suppress the development of cascades.

\section{ANALYTICAL TREATMENT}

The observables of the flare that enter our analysis are presented in Table 1. Following PD16, we derive the parameter values that minimize the jet power in the LHS scenario. These are summarized in Table 2 (Case A). In the following, the subscript "min" is used to denote quantities at minimum power conditions. The absolute minimum jet power is $P_{\mathrm{j}, \min } \simeq 14 \dot{M} c^{2}$ (see also Fig. 2 Ghisellini et al. 2014), where $\dot{M} c^{2} \simeq 10 L_{\mathrm{d}}$ for a geometrically thin and optically thick accretion disk with bolometric luminosity $L_{\mathrm{d}} \simeq 6 \times 10^{45} \mathrm{erg} \mathrm{s}^{-1}$ (Pian et al. 1999; Hayashida et al. 2015). At minimum power conditions, $B_{\min }^{\prime} \sim 2 \mathrm{kG}$ and the emitting region is in rough equipartition between magnetic fields and relativistic protons $\left(u_{\mathrm{p} \text {,min }}^{\prime} \sim u_{\mathrm{B} \text {, min }}^{\prime} \simeq 2 \times 10^{5} \mathrm{erg} \mathrm{cm}^{-3}\right)$. Henceforth, all primed quantities are measured in the rest frame of the emitting source, while unprimed quantities are measured in the observer's frame. In such strong magnetic fields, the energy of protons producing synchrotron photons at the peak energy of the $\gamma$-ray component is $E_{\mathrm{p}, \max }^{\prime} \sim 50 \mathrm{PeV}$. This is lower than the energy of protons that can be confined in the source by a factor $\sim 2400$ (Hillas 1984). Because of the short variability timescale and strong magnetic fields, the synchrotron cooling timescale of the highest energy protons in the source is comparable to the dynamical timescale $\left(t_{\text {syn }}^{\prime} \sim 3.5 t_{\text {dyn }}^{\prime} \simeq 2 \times 10^{4} \mathrm{~s}\right)$.

In the following, we assume that the size of the emitting source is approximately equal to the size of the dissipation region. De-
Table 2. Parameter values that minimize the jet power of $3 \mathrm{C} 279$ in the LHS scenario for the minute-scale GeV flare (Case A). Other values considered in $\$ 3$ are also listed (Case B). All primed quantities are measured in the rest frame of the emitting source. The beaming factor - defined as $\psi \equiv 2 \Gamma / \delta-$ was set equal to 1.01 .

\begin{tabular}{llll}
\hline Symbol & Parameter & Case A & Case B \\
\hline$\delta$ & Doppler factor & 19.5 & 50 \\
$\Gamma$ & Bulk Lorentz factor & 9.9 & 25 \\
$B^{\prime}(\mathrm{kG})$ & Magnetic field strength & 2.2 & 0.8 \\
$r_{\mathrm{b}}^{\prime}(\mathrm{cm})$ & Source radius & $1.7 \times 10^{14}$ & $4.4 \times 10^{14}$ \\
$P_{\mathrm{j}}\left(\mathrm{erg} \mathrm{s}^{-1}\right)$ & Absolute total jet power & $8.3 \times 10^{47}$ & $10^{48}$ \\
$u_{\mathrm{p}}^{\prime}\left(\mathrm{erg} \mathrm{cm}^{-3}\right)$ & Proton energy density & $2.6 \times 10^{5}$ & $7 \times 10^{3}$ \\
$u_{\mathrm{B}}^{\prime}\left(\mathrm{erg} \mathrm{cm}^{-3}\right)$ & Magnetic energy density & $2 \times 10^{5}$ & $2.6 \times 10^{4}$ \\
$E_{\mathrm{p}, \text { max }}^{\prime}(\mathrm{eV})$ & Max. proton energy & $5.2 \times 10^{16}$ & $4.8 \times 10^{16}$ \\
$z_{\mathrm{diss}}(\mathrm{cm})$ & Dissipation distance & $1.7 \times 10^{16}$ & $1.1 \times 10^{17}$ \\
\hline
\end{tabular}

pending on its opening angle $\theta$, the dissipation distance may lie from several up to hundreds of gravitational radii $\left(r_{\mathrm{g}}\right)$ away from the base of the jet. Assuming that $\theta \approx \theta_{\mathrm{obs}}$ and $\Gamma \theta_{\mathrm{obs}}=0.1$, we find $z_{\text {diss,min }} \simeq r_{\mathrm{b} \text {,min }}^{\prime} / \theta \simeq 143 r_{\mathrm{g}}<R_{\mathrm{BLR}}$. For a smaller degree of collimation (e.g., $\psi=2$ ) the dissipation distance would be placed at $28 r_{\mathrm{g}}$ from the central black hole, while the mimimum jet power would be $\psi^{2}$ times higher.

We next provide estimates of the efficiency for $p \gamma$ interactions and the optical depth for internal $\gamma \gamma$ absorption using simplified expressions for the respective cross sections. We will take into account the radiation from the illumination of the BLR as well as the internal synchrotron radiation produced by the relativistic electron and proton distributions; photons from the highenergy hump of the spectrum have not been taken into account in previous works (e.g., Dermer et al. 2007; Sikora et al. 2009). For the purposes of the analytical treatment, we model the internal target photon field as a broken power law, i.e., $n_{\mathrm{i}}^{\prime}\left(\epsilon^{\prime}\right)=$ $n_{\mathrm{i}, 0}^{\prime} / \epsilon^{\prime 2}\left[\left(\epsilon^{\prime} / \epsilon_{\mathrm{i}}^{\prime}\right)^{\alpha_{\mathrm{i}}} H\left(1-\epsilon^{\prime} / \epsilon_{\mathrm{i}}^{\prime}\right)+\left(\epsilon^{\prime} / \epsilon_{\mathrm{i}}^{\prime}\right)^{\beta_{\mathrm{i}}} H\left(\epsilon^{\prime} / \epsilon_{\mathrm{i}}^{\prime}-1\right)\right]$, where $\epsilon^{\prime}$ is the photon energy in $m_{\mathrm{e}} c^{2}$ units, $H(x)$ is the Heavyside function, $\alpha_{\mathrm{i}}>0, \beta_{\mathrm{i}} \leqslant 0, \epsilon_{\mathrm{i}}^{\prime}=\epsilon_{\mathrm{i}}(1+z) / \delta$, and $\mathrm{i}=\mathrm{e}(\mathrm{p})$ for electron (proton) synchrotron radiation. In particular, $\epsilon_{\mathrm{p}}=E_{\gamma} / m_{\mathrm{e}} c^{2}$ with $E_{\gamma}=0.7 \mathrm{GeV}$ (Table 1) and $\epsilon_{\mathrm{e}}=8 \times 10^{-9} v_{12}$, where we introduced the notation $Q_{x} \equiv Q / 10^{x}$ in cgs units. Our choice for the low peak energy is based on archival observations (see also Fig. 1). The normalization of the photon number density is given by $n_{\mathrm{i}, 0}^{\prime}=3 L_{\mathrm{i}} /\left(4 \pi r_{\mathrm{b}}^{\prime 2} \delta^{4} m_{\mathrm{e}} c^{3}\right)$, where $r_{\mathrm{b}}^{\prime}=c \delta t_{\mathrm{v}} /(1+z), L_{\mathrm{e}} \equiv L$, and $L_{\mathrm{p}} \equiv L_{\gamma}$ (Table 1$)$. The BLR energy density profile is assumed to be uniform up to a typical radius $R_{\text {BLR }}$ (e.g., Ghisellini \& Tavecchio 2008; Dermer et al. 2014). Its differential photon number density is approximated by $n_{\mathrm{BLR}}^{\prime}\left(\epsilon^{\prime}\right)=$ $15 u_{\mathrm{BLR}}^{\prime}\left(m_{\mathrm{e}} c^{2}\right)^{3} \epsilon^{\prime 2}\left(e^{\epsilon^{\prime} m_{\mathrm{e}} c^{2} / k T^{\prime}}-1\right)^{-1} /\left(\pi k T^{\prime}\right)^{4}$, where $k T^{\prime} \simeq \Gamma E_{\mathrm{BLR}}$, $E_{\mathrm{BLR}}=10 \mathrm{eV}, u_{\mathrm{BLR}}^{\prime} \approx \Gamma^{2} L_{\mathrm{BLR}} / 4 \pi c R_{\mathrm{BLR}}^{2}$, and $L_{\mathrm{BLR}} \simeq 6 \times 10^{44} \mathrm{erg}$ $\mathrm{s}^{-1}$ (Celotti et al. 1997). At $z_{\text {diss,min }}$ the direct disk radiation can be neglected, since $u_{\mathrm{d}}^{\prime} / u_{\mathrm{BLR}}^{\prime} \simeq 0.08$. Here, we assumed that the size of the accretion disk that emits most of the bolometric radiation is $R_{\mathrm{d}}=10^{15} \sim 10 r_{\mathrm{g}}$ (Ghisellini \& Madau 1996; Sikora et al. 2009).

The energy loss timescale due to $\mathrm{p} \gamma$ interactions is given by $t_{p \gamma}^{\prime-1}\left(\gamma_{\mathrm{p}}^{\prime}\right)=c /\left(2 \gamma_{\mathrm{p}}^{\prime 2}\right) \int_{\bar{\epsilon}_{\mathrm{th}}}^{\infty} \mathrm{d} \bar{\epsilon} \sigma_{\mathrm{p} \gamma}(\bar{\epsilon}) \kappa_{\mathrm{p} \gamma}(\bar{\epsilon}) \bar{\epsilon} \int_{\bar{\epsilon} / 2 \gamma_{\mathrm{p}}^{\prime}}^{\infty} \mathrm{d} \epsilon^{\prime} n^{\prime}\left(\epsilon^{\prime}\right) / \epsilon^{\prime 2}$ (Stecker 1968; Sikora et al. 1987), where $\gamma_{\mathrm{p}}^{\prime}$ is the proton's Lorentz factor, bared quantities are measured in the proton's rest frame, $\bar{\epsilon}_{\mathrm{th}} \simeq 300$, $n^{\prime}\left(\epsilon^{\prime}\right)$ is the number density of target photons. In the analytical calculations we use $\sigma_{\mathrm{p} \gamma} \approx 0.1 \mathrm{mb} H\left(\bar{\epsilon}-\bar{\epsilon}_{\mathrm{th}}\right)$, and $\kappa_{\mathrm{p} \gamma}=0.2$. The exact cross section, inelasticity and pion multiplicity are used in the numerical calculations (Dimitrakoudis et al. 2012). The efficiency for 
$\mathrm{p} \gamma$ interactions, defined as $\eta_{\mathrm{p} \gamma} \equiv r_{\mathrm{b}}^{\prime} / c t_{\mathrm{p} \gamma}^{\prime}$, on electron synchrotron photons $\left(\alpha_{\mathrm{e}}>1\right.$ and $\left.\beta_{\mathrm{e}}<0\right)$ for $\gamma_{\mathrm{p}}^{\prime} \ll 10^{11} \delta_{1} / \epsilon_{\mathrm{e},-8}$ is

$\eta_{\mathrm{p} \gamma, \mathrm{e}} \simeq 1.4 \times 10^{5} \frac{L_{47} t_{v, 2}^{-1} \delta_{1}^{-4} \epsilon_{\mathrm{e},-8}^{-1}}{\left(1-\beta_{\mathrm{e}}\right)\left(3-\beta_{\mathrm{e}}\right)}\left(\frac{10^{11} \delta_{1}}{\gamma_{\mathrm{p}}^{\prime} \epsilon_{\mathrm{e},-8}}\right)^{-1+\beta_{\mathrm{e}}}$.

For parameters that minimize the jet power (Table 2) and $\beta_{\mathrm{e}} \sim$ $-1 / 4$, we find $\eta_{\mathrm{p} \gamma \mathrm{e}} \ll 1$. The efficiency also depends strongly on the Doppler factor as $\eta_{\mathrm{p} \gamma, \mathrm{e}} \propto \delta^{-5+\beta_{\mathrm{e}}}$. For $\mathrm{p} \gamma$ interactions on proton synchrotron photons $\left(0<\alpha_{\mathrm{p}}<1\right.$ and $\left.\beta_{\mathrm{p}}<0\right)$ and $\gamma_{\mathrm{p}}^{\prime} \gg \delta_{1} / \epsilon_{\mathrm{p}, 3}$ we find

$\eta_{\mathrm{p} \gamma, \mathrm{p}}=1.3 \times 10^{-4} \frac{L_{49} t_{v, 2}^{-1} \delta_{1}^{-4} \epsilon_{\mathrm{p}, 3}^{-1}}{\left(1-\alpha_{\mathrm{p}}\right)\left(3-\alpha_{\mathrm{p}}\right)}\left(\frac{2 \gamma_{\mathrm{p}}^{\prime} \epsilon_{\mathrm{p}, 3}}{\delta_{1}}\right)^{1-\alpha_{\mathrm{p}}}$.

The efficiency increases with increasing proton energy as $\gamma_{\mathrm{p}}^{\prime 1-\alpha_{\mathrm{p}}}$ and $\eta_{\mathrm{p} \gamma, \mathrm{p}} \propto \delta^{-5+\alpha_{\mathrm{p}}}$, where $\alpha_{\mathrm{p}}$ corresponds to the X-ray-to- $\gamma$-ray photon index of the spectrum in the LHS model. For $\mathrm{p} \gamma$ interactions with BLR photons from the Rayleigh-Jeans part of the spectrum, the efficiency is independent of the proton's energy and is given by

$\eta_{\mathrm{p} \gamma, \mathrm{BLR}} \simeq 2 \times 10^{-5} \frac{t_{v, 2} L_{\mathrm{BLR}, 45} \delta_{1}^{2}}{\epsilon_{\mathrm{BLR},-5} R_{\mathrm{BLR}, 17}^{2}}, \gamma_{\mathrm{p}}^{\prime} \gg \frac{9 \times 10^{6}}{\psi \delta_{1} \epsilon_{\mathrm{BLR},-5}}$.

In contrast to $\eta_{\mathrm{p} \gamma, \mathrm{e}(\mathrm{p})}$ that have a strong inverse dependence on the Doppler factor, $\eta_{\mathrm{p} \gamma, \mathrm{BLR}} \propto \delta^{2}$. The optical depth for internal $\gamma \gamma$ absorption is $\tau_{\gamma \gamma}\left(\epsilon_{1}\right)=r_{\mathrm{b}}^{\prime} \int \mathrm{d} \epsilon^{\prime} n^{\prime}\left(\epsilon^{\prime}\right) \sigma_{\gamma \gamma}\left(\epsilon^{\prime}, \epsilon_{1}^{\prime}\right)$, where $\sigma_{\gamma \gamma}\left(\epsilon^{\prime}, \epsilon_{1}^{\prime}\right)=$ $0.652 \sigma_{\mathrm{T}} H\left(\epsilon_{1}^{\prime} \epsilon^{\prime}-2\right) /\left(\epsilon_{1}^{\prime} \epsilon^{\prime}\right)$ (e.g., Coppi \& Blandford 1990). The respective optical depth due to electron synchrotron photons may be written as

$\tau_{\gamma \gamma, \mathrm{e}}\left(\epsilon_{1}^{\prime}\right) \simeq \frac{6 \times 10^{7+\beta_{\mathrm{e}}}(1+z)^{1-\beta_{\mathrm{e}}}}{2^{2-\beta_{\mathrm{e}}}\left(2-\beta_{\mathrm{e}}\right)} \frac{L_{47} \epsilon_{1,8}^{\prime 1-\beta_{\mathrm{e}}}}{t_{\mathrm{v}, 2} \delta_{1}^{5-\beta_{\mathrm{e}}} \epsilon_{\mathrm{e},-8}^{\beta_{\mathrm{e}}}}, \epsilon_{1}^{\prime} \ll 10^{9} \frac{\delta_{1}}{\epsilon_{\mathrm{e},-8}}$.

In the above, we used $\alpha_{\mathrm{e}}>2$, since the electron synchrotron spectrum below its peak is usually synchrotron self-absorbed (see Fig. 1 below). Noting that $0<\alpha_{\mathrm{p}}<1$, the optical depth due to internal absorption on proton synchrotron photons is given by

$\tau_{\gamma \gamma, \mathrm{p}}\left(\epsilon_{1}^{\prime}\right) \simeq \frac{(1+z)^{1-\alpha_{\mathrm{p}}}}{2^{2-\alpha_{\mathrm{p}}}\left(2-\alpha_{\mathrm{p}}\right)} \frac{L_{\gamma, 49} \epsilon_{1,-2}^{\prime 1-\alpha_{\mathrm{p}}}}{t_{v, 2} \epsilon_{\mathrm{p}, 3}^{\alpha_{\mathrm{p}}} \delta^{5-\alpha_{\mathrm{p}}}}, \epsilon_{1}^{\prime} \gg 10^{-2} \frac{\delta_{1}}{\epsilon_{\mathrm{p}, 3}}$.

The optical depth increases for increasing photon energy as $\tau_{\gamma \gamma, \mathrm{p}} \propto$ $\epsilon_{1}^{\prime 1-\alpha_{\mathrm{p}}}$, while it depends on the Doppler factor as $\delta^{-5+\alpha_{\mathrm{p}}}$. Substitution of parameter values that minimize the jet power (see Table 2) and of the flare's observables (Table 1) in eqs. (4) and (5) shows that any internally produced radiation at $E \gtrsim 20 \mathrm{GeV}$ will be attenuated by the electron and proton synchrotron radiation fields. In fact, an EMC can be developed, since $\tau_{\gamma \gamma} \gg 1$ at multi-TeV energies. Depending on the luminosity of the multi-TeV photons and the exact value of $\tau_{\gamma \gamma}$, the spectrum of photons originally considered as targets will be modified. We expand on this issue in $\S 3$ with detailed numerical calculations. Finally, the optical depth due to the Rayleigh-Jeans part of the BLR photon energy spectrum may be written as

$\tau_{\gamma \gamma, \mathrm{BLR}} \simeq 0.6 \frac{\delta_{1} t_{\mathrm{v}, 2} L_{\mathrm{BLR}, 45}}{\epsilon_{1,5}^{\prime} \epsilon_{\mathrm{BLR},-5}^{2} R_{\mathrm{BLR}, 17}^{2}}, \epsilon_{1}^{\prime} \gg \frac{10^{5}}{\psi \delta_{1} \epsilon_{\mathrm{BLR},-5}}$.

In contrast to $\tau_{\gamma \gamma, \mathrm{e}(\mathrm{p})}$ which decrease with increasing $\delta$ (eqs. (4) and (5)) the respective optical depth due to the BLR radiation field is $\propto \delta$ as long as the $\gamma$-ray emitting region lies within the BLR.

\section{NUMERICAL SED MODELLING}

Using as a starting point the parameter values of Case A (Table 2), we perform time-dependent calculations of the broad-band spec-

(C) 2016 RAS, MNRAS 000, 1-??
Table 3. Parameters of the particle distributions at injection as derived from the numerical modelling of the flare's time-averaged spectrum over the orbit $\mathrm{D}$ for both cases shown in Fig. 1.

\begin{tabular}{cccccccc}
\hline$\gamma_{\mathrm{p}, \min }^{\prime}$ & $\gamma_{\mathrm{p}, \mathrm{br}}^{\prime}$ & $\gamma_{\mathrm{p}, \max }^{\prime}$ & $s_{\mathrm{p}, 1}$ & $s_{\mathrm{p}, 2}$ & $\gamma_{\mathrm{e}, \min }^{\prime}$ & $\gamma_{\mathrm{e}, \max }^{\prime}$ & $s_{\mathrm{e}}$ \\
1 & $10^{6}$ & $10^{8}$ & 1.6 & 2.1 & 1 & 160 & 2.1 \\
\hline
\end{tabular}

trum aiming at fitting the average spectrum of the outburst phase from orbit D (Ackermann et al. 2016). Simultaneous observational data were available in the UV, X-ray and $\gamma$-ray bands, but not in the radio-to-optical bands. The latter are not crucial for our analysis, as our main conclusions remain unaffected by a different modelling of the low-energy component of the SED. Although a detailed fit to the light curve during Orbit D lies beyond the scope of this letter, we do take into account the basic temporal properties of the flare (i.e., duration, peak and average fluxes). In an attempt to model the flare's broad-band spectrum with the minimum number of changes in the model parameters, we imposed fluctuations on the proton energy density alone: $u_{\mathrm{p}}^{\prime}(\tau)=\tilde{u}_{\mathrm{p}}^{\prime}\left[1+4.75 /\left(0.25+(\tau-5)^{2}\right)\right]$, where $\tau \equiv t^{\prime} / t_{\mathrm{dyn}}^{\prime}=[0,10]$ and $\tilde{u}_{\mathrm{p}}^{\prime}=8 \times 10^{4}(800) \mathrm{erg} \mathrm{cm}^{-3}$ for Case A (Case B). The proton energy density averaged over ten dynamical timescales is $\left\langle u_{\mathrm{p}}^{\prime}\right\rangle=3 \times 10^{5}\left(3 \times 10^{3}\right) \mathrm{erg} \mathrm{cm}^{-3}$ for Case A (Case B), in agreement with our analytical estimates in Table 2. In both cases, we choose initial conditions that correspond to a low flux level of the source. In the numerical calculations we also lift some of the simplifying assumptions (e.g., monoenergetic proton distribution) used in PD16 and §2. In particular, the particle injection spectra are modelled as broken power laws, i.e, $n_{\mathrm{i}}^{\prime}\left(\gamma_{\mathrm{i}}^{\prime}\right) \propto$ $\gamma_{\mathrm{i}}^{\prime-s_{\mathrm{i}, 1}} S\left(\gamma_{\mathrm{i}}^{\prime} ; \gamma_{\mathrm{i}, \min }^{\prime}, \gamma_{\mathrm{i}, \mathrm{br}}^{\prime}\right)+\gamma_{\mathrm{p}}^{\prime-s_{\mathrm{i}, 2}} S\left(\gamma_{\mathrm{p}}^{\prime} ; \gamma_{\mathrm{i}, \mathrm{br}}^{\prime}, \gamma_{\mathrm{i}, \max }^{\prime}\right)$, where $S\left(y ; y_{1}, y_{2}\right)$ is the unit boxcar function. The parameter values derived from the fits are presented in Table 3. Because of the derived $\mathrm{kG}$ magnetic fields, our numerical calculations also include muon and pion losses due to synchrotron radiation (Petropoulou et al. 2014).

Our results are shown in Fig. 1. In each panel, we present the 0.1-10 GeV light curves (top) and the broad-band photon spectra (bottom); for more details, see figure caption. The results obtained by neglecting (taking into account) internal $\gamma \gamma$ absorption are plotted, in both panels, with black (light blue) lines. For parameters that minimize the jet power (left panel), an EMC is being developed with a photon spectrum that cannot describe the observed one. Photons produced via photohadronic processes (at multi-TeV energies) are attenuated and their luminosity is transfered to lower (i.e., IR-to-X-rays) energies. The increase of the target photon number density in those softer bands increases, in turn, the efficiency for photohadronic interactions, thus leading to an increased production rate of VHE photons. This creates a feedback loop of processes that results in higher photon luminosities and spectral shapes dramatically different than the target photon spectra before the initiation of the EMC (see also Petropoulou \& Mastichiadis 2012; Petropoulou 2014). Because of the lower peak photon energy (few MeV) of the EMC spectrum, the model light curve in the 0.1-10 GeV lies far below the observed fluxes (left top panel in Fig. 1). We next seeked for parameters that would (i) provide a successful fit to the data from Orbit D, (ii) suppress the development of EMC, and (iii) lead to $P_{\mathrm{j}} \gtrsim P_{\mathrm{j}, \min }$ (Case B). The EMC is suppressed compared to Case A, but the internal absorption of VHE photons still leads to an excess below few $\mathrm{keV}$. Because of the higher $\delta$, the $\mathrm{p} \gamma$ photon production in Case B is driven by the BLR radiation field ( $\$ 2)$. 

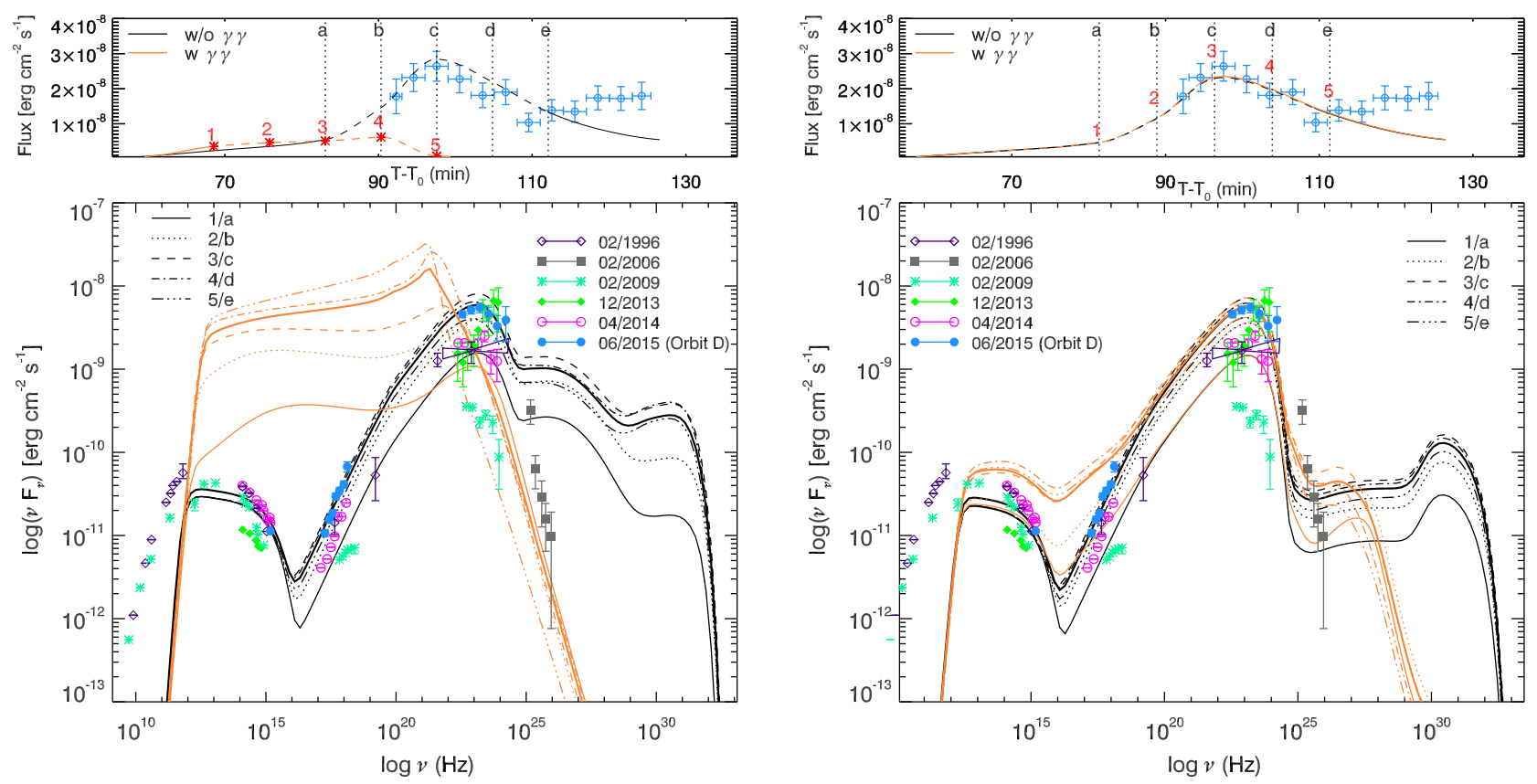

Figure 1. The 3-minute binned Fermi-LAT light curve from Orbit D (top panels). Here, $T_{0}$ is defined as June 16 2015 02:00:00 (UT). For the conversion from photon to energy fluxes, a power-law spectrum with photon index -2.1 in the range $0.1-10 \mathrm{GeV}$ was assumed. The model light curves with (orange) and without (black) $\gamma \gamma$ absorption are overplotted. The dashed part of the light curves indicates the period where the time-averaging of the model spectra has been performed. Vertical lines and asterisks denote the times of the model spectra presented in the bottom panel. SED of 3C 279 compiled using UV, X-ray and Fermi-LAT data of Orbit D (blue filled circles) during the latest outburst on June 2015 (bottom panels). Multi-wavelength data of past flaring periods (adopted by Ackermann et al. (2016)) are also shown with coloured symbols (see legend). SED snapshots during the outburst phase from Orbit D are presented with thin lines. The numbering and color coding of the curves follows the notation in the top panel. Thick lines show the model spectra averaged over a period of $\sim 30 \mathrm{~min}$ (dashed lines on top panel). The displayed spectra do not take into account the attenuation on the extragalactic background light. Left panel: The parameters are chosen as to fit the average spectrum of Orbit D and to minimize the jet power (Case A in Table 2). Right panel: The parameters used (Case B) correspond to $\delta \simeq 2.5 \delta_{\min }, B^{\prime}=0.4 B_{\min }^{\prime}, P_{\mathrm{j}}=1.25 P_{\mathrm{j} \text {,min }}$, and $z_{\mathrm{diss}} \simeq R_{\mathrm{BLR}}$.

\section{DISCUSSION}

We have derived parameter values that minimize the jet power of 3C 279 during the minute-scale Fermi-LAT flare in the LHS model. For these conditions, however, an EMC is developed in the $\gamma$-ray emitting region due to the internal absorption of multi- $\mathrm{TeV}$ photons produced via photohadronic interactions. The most straightforward way for suppressing the development of an EMC is to decrease the number of relativistic pairs that are being injected in the emitting region by $\mathrm{p} \gamma$ and $\gamma \gamma$ processes. This can be achieved in a faster moving emitting region because of the strong dependence of $\eta_{\mathrm{p} \gamma, \mathrm{e}(\mathrm{p})}$ and $\tau_{\gamma \gamma, \mathrm{e}(\mathrm{p})}$ on $\delta(\S 2)$. To ensure that $P_{\mathrm{j}} \gtrsim P_{\mathrm{j}, \mathrm{min}}$, the magnetic field in the emitting region should be $B^{\prime}<B_{\min }^{\prime}$ for $\delta>\delta_{\min }$, given that the main contributing terms to the total jet power scale as $P_{\mathrm{j}, \mathrm{B}} \propto B^{\prime 2} \delta^{4}$ and $P_{\mathrm{j}, \mathrm{p}} \propto B^{\prime-6} \delta^{-6}$. Taking also into account the radiation power, we find that $P_{\mathrm{j}} \simeq 1.25 P_{\mathrm{j}, \min }$ for $\delta=2.5 \delta_{\min } \simeq 50$ and $B^{\prime}=0.8 \mathrm{kG}$ (Table 2). We have shown that a fit to the $\gamma$-ray spectrum of the flare is possible for this parameter set (Case B). However, our model spectra cannot describe well the observations below few $\mathrm{keV}$ due to the EMC emission. The discrepancy between the data and the model could be reduced, if $u_{\mathrm{BLR}}^{\prime}$ was lower by a factor of ten than the adopted value. This would, in turn, suggest a more extended BLR $\left(R_{\mathrm{BLR}}>0.25 \mathrm{pc}\right)$ than the one predicted by the simple scaling relation of Ghisellini \& Tavecchio (2008).

For a BLR with properties as those listed in Table 1, the effects of the EMC on the broad-band spectrum could be suppressed, if the $\gamma$-ray emitting region was located outside the BLR (see Nalewajko et al. 2014, for leptonic scenarios). This would re- quire $\delta \gg \sqrt{2 R_{\mathrm{BLR}}(1+z) \sqrt{\psi-1} / c t_{\mathrm{v}} \psi} \simeq 70$ for $\Gamma \theta_{\mathrm{obs}}=0.1$. The jet power could still be close to the minimum one provided that $B^{\prime}>100 \mathrm{G}$ at distances $>0.1 \mathrm{pc}$ (see also Bottacini et al. 2016). The main challenge is to explain then, the extremely high local energy densities (Nalewajko et al. 2012) associated with the strong magnetic fields. Such regions in parsec-scale jets would be severely over-pressured, and would not survive for more than a couple of dynamical time scales. Were the magnetic field strength lower (e.g., $10 \mathrm{G}$ ), the energetic requirements would be very high, i.e., $P_{\mathrm{j}} \gg 100 \dot{M} c^{2}$ (e.g. Petropoulou \& Dimitrakoudis 2015).

It is noteworthy that a flat proton energy distribution $\left(s_{\mathrm{p}} \lesssim 2\right)$ is required for explaining the high-energy hump of the SED (see also Böttcher et al. 2013; Cerruti et al. 2015), while $s_{\mathrm{e}} \sim 2$ (see Table 3). Magnetic reconnection in highly magnetized plasmas may account for flat particle energy spectra, as demonstrated by particlein-cell (PIC) simulations in electron-positron plasmas for $\sigma \gtrsim$ 10 (Guo et al. 2014; Sironi \& Spitkovsky 2014; Nalewajko et al. 2015). Similar behavior is expected in electron-proton plasmas for $\sigma \gg 1$ (Melzani et al. 2014; Guo et al. 2016), where $\sigma=$ $B_{0}^{2} /\left(4 \pi \rho_{0} c^{2}\right)$ is the jet magnetization, $B_{0}$, and $\rho_{0} c^{2}$ are the unreconnected magnetic field strength and plasma rest mass energy density, respectively. The latter can be estimated from the energy density at the blazar dissipation region, $u_{\mathrm{j}}^{\prime} \sim P_{\mathrm{j}} \epsilon_{\mathrm{r}}^{2} /\left(2 \pi c \Gamma^{6} \theta^{2} r_{\mathrm{g}}^{2}\right)$, where $\epsilon_{\mathrm{r}}=0.1 \epsilon_{r,-1}$ is the reconnection rate (see also Giannios 2013). The jet's magnetic field strength far from the reconnection layer can be estimated from the magnetic field in the emission region as $B_{0} \sim B^{\prime} / \sqrt{2}$ (Sironi et al. 2016). For typical parameters, we find that $B_{0}^{2} /(8 \pi) \sim \rho_{0} c^{2} \sim u_{\mathrm{j}}^{\prime} / 2$ or equivalently $\sigma \sim 1$. As PIC sim- 
ulations of reconnection in moderately magnetized electron-proton plasmas are sparse, the properties of the accelerated particle distributions in this regime remain unclear (Dr. Sironi, private communication).

The minute-scale variability and high $\gamma$-ray luminosity characterizing the 2015 outburst from 3C 279 challenge both leptonic and leptohadronic one-zone models for the blazar emission. Allowing for relativistic motion of the emitting region in the rest frame of the jet, as proposed in models of magnetic reconnection (e.g., Giannios et al. 2009; Nalewajko et al. 2011; Petropoulou et al. 2016), might resolve some of the issues that single-zone models encounter.

\section{ACKNOWLEDGMENTS}

We thank the anonymous referee for an insightful report and Prof. M. Sikora for useful comments on the manuscript. M. P. acknowledges support from NASA through the Einstein Postdoctoral Fellowship grant number PF3 140113 awarded by the Chandra X-ray Center, which is operated by the Smithsonian Astrophysical Observatory for NASA under contract NAS8-03060. K.N. was supported by the Polish National Science Centre grant 2015/18/E/ST9/00580.

\section{REFERENCES}

Ackermann M. et al., 2016, Astrophysical Journal Letters, 824, L20

Aharonian F. et al., 2007, Astrophysical Journal Letters, 664, L71

Aharonian F. A., 2000, New Astron., 5, 377

Albert J. et al., 2007, Astrophysical Journal Letters, 667, L21

Aleksić J. et al., 2011, Astrophysical Journal Letters, 730, L8

Asano K., Hayashida M., 2015, Astrophysical Journal Letters, 808, L18

Bottacini E., Boettcher M., Pian E., Collmar W., 2016, ArXiv eprints (1610.01617)

Böttcher M., Reimer A., Sweeney K., Prakash A., 2013, Astrophysical Journal, 768, 54

Celotti A., Padovani P., Ghisellini G., 1997, Monthly Notices of the Royal Astronomical Society, 286, 415

Cerruti M., Zech A., Boisson C., Inoue S., 2015, Monthly Notices of the Royal Astronomical Society, 448, 910

Coppi P. S., Blandford R. D., 1990, Monthly Notices of the Royal Astronomical Society, 245, 453

Dermer C. D., Cerruti M., Lott B., Boisson C., Zech A., 2014, Astrophysical Journal, 782, 82

Dermer C. D., Ramirez-Ruiz E., Le T., 2007, Astrophysical Journal Letters, 664, L67

Dermer C. D., Schlickeiser R., Mastichiadis A., 1992, Astronomy \& Astrophysics, 256, L27

Dimitrakoudis S., Mastichiadis A., Protheroe R. J., Reimer A., 2012, Astronomy \& Astrophysics, 546, A120

Dimitrakoudis S., Petropoulou M., Mastichiadis A., 2014, Astroparticle Physics, 54, 61

Fortson L., VERITAS Collaboration, Fermi-LAT Collaborators, 2012, in American Institute of Physics Conference Series, Vol. 1505, American Institute of Physics Conference Series, Aharonian F. A., Hofmann W., Rieger F. M., eds., pp. 514-517

Ghisellini G., Madau P., 1996, Monthly Notices of the Royal Astronomical Society, 280, 67

Ghisellini G., Tavecchio F., 2008, Monthly Notices of the Royal Astronomical Society, 387, 1669
Ghisellini G., Tavecchio F., Maraschi L., Celotti A., Sbarrato T., 2014, Nature, 515, 376

Giannios D., 2013, Monthly Notices of the Royal Astronomical Society, 431, 355

Giannios D., Uzdensky D. A., Begelman M. C., 2009, Monthly Notices of the Royal Astronomical Society, 395, L29

Guo F., Li H., Daughton W., Liu Y.-H., 2014, Physical Review Letters, 113, 155005

Guo F. et al., 2016, Astrophysical Journal Letters, 818, L9

Hayashida M. et al., 2015, Astrophysical Journal, 807, 79

Hillas A. M., 1984, Ann. Rev. Astron. Asrophys., 22, 425

Khangulyan D. V., Barkov M. V., Bosch-Ramon V., Aharonian F. A., Dorodnitsyn A. V., 2013, Astrophysical Journal, 774, 113 Lynds C. R., Stockton A. N., Livingston W. C., 1965, Astrophysical Journal, 142, 1667

Mannheim K., Biermann P. L., Kruells W. M., 1991, Astronomy \& Astrophysics, 251, 723

Melzani M., Walder R., Folini D., Winisdoerffer C., Favre J. M., 2014, Astronomy \& Astrophysics, 570, A112

Mücke A., Protheroe R. J., 2001, Astroparticle Physics, 15, 121

Nalewajko K., Begelman M. C., Cerutti B., Uzdensky D. A., Sikora M., 2012, Monthly Notices of the Royal Astronomical Society, 425, 2519

Nalewajko K., Giannios D., Begelman M. C., Uzdensky D. A., Sikora M., 2011, Monthly Notices of the Royal Astronomical Society, 413, 333

Nalewajko K., Sikora M., Begelman M. C., 2014, Astrophysical Journal Letters, 796, L5

Nalewajko K., Uzdensky D. A., Cerutti B., Werner G. R., Begelman M. C., 2015, Astrophysical Journal, 815, 101

Petropoulou M., 2014, Monthly Notices of the Royal Astronomical Society, 442, 3026

Petropoulou M., Dermer C. D., 2016, Astrophysical Journal Letters, $825, \mathrm{~L} 11$

Petropoulou M., Dimitrakoudis S., 2015, Monthly Notices of the Royal Astronomical Society, 452, 1303

Petropoulou M., Giannios D., Dimitrakoudis S., 2014, Monthly

Notices of the Royal Astronomical Society, 445, 570

Petropoulou M., Giannios D., Sironi L., 2016, Monthly Notices of the Royal Astronomical Society, 462, 3325

Petropoulou M., Mastichiadis A., 2012, Monthly Notices of the Royal Astronomical Society, 421, 2325

Pian E. et al., 1999, Astrophysical Journal, 521, 112

Sikora M., Begelman M. C., Rees M. J., 1994, Astrophysical Journal, 421, 153

Sikora M., Kirk J. G., Begelman M. C., Schneider P., 1987, Astrophysical Journal Letters, 320, L81

Sikora M., Stawarz Ł., Moderski R., Nalewajko K., Madejski G. M., 2009, Astrophysical Journal, 704, 38

Sironi L., Giannios D., Petropoulou M., 2016, Monthly Notices of the Royal Astronomical Society, 462, 48

Sironi L., Spitkovsky A., 2014, Astrophysical Journal Letters, 783, L21

Stecker F. W., 1968, Physical Review Letters, 21, 1016

Zdziarski A. A., Böttcher M., 2015, Monthly Notices of the Royal Astronomical Society, 450, L21 